\title{
ADMINISTRAÇÃO DE MEDICAMENTOS: UMA VISÃO SISTÊMICA PARA O DESENVOLVIMENTO DE MEDIDAS PREVENTIVAS DOS ERROS NA MEDICAÇÃO
}

\author{
DRUG ADMINISTRATION: A SYSTEMIC VIEW FOR THE DEVELOPMENT OF MEDICATION ERROR \\ PREVENTIVE ACTIONS \\ ADMINISTRACIÓN DE MEDICAMENTOS: UNA VISIÓN SISTÉMICA PARA EL DESARROLLO DE MEDIDAS \\ PREVENTIVAS DE ERRORES EN LA MEDICACIÓN
}

\section{Ana Elisa Bauer de Camargo Silva ${ }^{1}$ Silvia Helena De Bortoli Cassiani ${ }^{2}$}

RESUMO: A administração de medicamentos é uma atividade freqüente para a enfermagem e dentro do ambiente hospitalar, porém estudos vêm demonstrando que erros nessa prática vem ocorrendo e que estratégias precisam ser implementadas no intuito de preveni-los ou minimizá-los. Possuir uma visão sistêmica possibilita a identificação dos pontos frágeis dos processos e o desenvolvimento de medidas que garantam maior segurança para o paciente e para os profissionais.

PALAVRAS-CHAVE: Erros de medicação; Sistemas de medicação; Sistemas de medicação no hospital

ABSTRACT: The drug administration is a very frequent activity of a hospital routine in general, as well as of its nursing team. However studies have shown the need to implement strategies to prevent or to mitigate errors found in this pratice. To look at the medication process thru a systemic view allows to identify its weak points and to develop procedures to assure the safety of the customers and the professionals.

KEY WORDS: Medication errors. Medication system; Medication system; hospital

RESUMEN: La administración de medicamentos es una actividad común para enfermería y dentro del ambiente hospitalario, sin embargo hay estudios que han demostrado errores que vienen ocurriendo en dicha práctica y las estrategias que necesitan ser implementadas en el intento de prevenirlos y minimizarlos. Poseer una visión sistémica de la medicación, posibilita la identificación de los puntos frágiles de los procesos y el desarrollo de medidas que garanticen mayor seguridad para el paciente y para los profesionales.

PALABRAS-CLAVE: Errores de medicación; Sistemas de medicación; Sistemas de medicación en el hospital.

\section{INTRODUÇÃO}

\section{ADMINISTRAÇÃO DE MEDICAMENTOS}

A administração de medicamentos, prática realizada nas instituições hospitalares sob responsabilidade da equipe de enfermagem, deve ser vista por todos os profissionais de saúde envolvidos com a terapia medicamentosa como apenas uma das partes do processo de medicação.

\footnotetext{
${ }^{1}$ Enfermeira. Mestre em Enfermagem. Professora Assistente da Faculdade de Enfermagem da Universidade Federal de Goiás. Rua 227, s/n, Qd. 68 - Setor leste Universitário - Goiânia -GO - CEP: 74605-080.

E-mail: anaelisa@terra.com.br 062 - 5211822 Fax: 5211807.

2 Enfermeira. Doutora em Enfermagem. Professora Associada do Departamento de Enfermagem Geral e Especializada da EERP - USP. shbcassi@eerp.usp.br.
} 
SILVA, Ana Elisa Bauer de Camargo; CASSIANI, Silvia Helena De Bortoli - Administração de medicamentos: uma visão sistêmica para o desenvolvimento de medidas preventivas dos erros na medicação. Revista Eletrônica de Enfermagem, v. 06, n. 02, p. 279-285, 2004. Disponível em www.fen.ufg.br

No que diz respeito à enfermagem esta deve ater-se não somente aos procedimentos técnicos e básicos inerentes à profissão, mas identificar os caminhos percorridos pelo medicamento desde o momento que o médico o prescreve até a sua administração ao paciente e analisar criticamente o sistema de medicação, refletindo sobre suas possíveis falhas e causas. A enfermagem deve colaborar com a segurança do sistema buscando soluções para os problemas existentes, além de colaborar com pesquisas sobre esta temática.

A utilização de medicamentos é uma das intervenções mais utilizadas no ambiente hospitalar, no entanto, estudos, ao longo dos últimos anos, têm evidenciado a presença de erros no tratamento medicamentoso causando prejuízos aos pacientes que vão desde o não-recebimento do medicamento necessário até lesões e mortes (LEAPE et al. 1995; TÁXIS \& BARBER, 2003). A administração de medicamentos corresponde a última oportunidade de prevenir um erro na medicação que pode ter surgido já na prescrição ou na dispensação dos medicamentos.

Os profissionais de saúde devem estar cientes e alertas para este fato e buscar, permanentemente, medidas de prevenção de erros através de novos conhecimentos, condutas ou de estratégias que visem proteger todos os envolvidos, principalmente o paciente.

Obter uma visão ampla do sistema de medicação possibilita aos profissionais condições de análise e intervenções que garantam uma assistência responsável e segura ao paciente e a si próprio. A partir dessas considerações esse estudo tem por objetivo discorrer sobre tipos e causas de erros na medicação e sobre a importância de que haja uma visão ampla do sistema de medicação dentro da instituição hospitalar.

\section{ERROS NA MEDICAÇÃO}

$\begin{array}{crrr}\text { Os erros na medicação são } & \text { ad } \\ \text { considerados } & \text { eventos } & \text { adversos } & \text { ao } \\ \text { medicamento passíveis } & \text { de prevenção, }\end{array}$

podendo ou não causar dano ao paciente, com possibilidade de ocorrer em um ou em vários momentos dentro do processo de medicação (BATES et al., 1995;. LEAPE et al. 1995).

O National Coordinating Council for Medication Error Reporting and Prevention (NCCMERP), corporação norte - americana independente, composta de 15 organizações apresenta as seguintes definições para dano e erro na medicação:

"Dano é definido como prejuízo temporário ou permanente da função ou estrutura do corpo: física, emocional, ou psicológica, seguida ou não de dor, requerendo uma intervenção".

"Erro na medicação é qualquer evento evitável que pode causar ou induzir ao uso inapropriado de medicamento ou prejudicar 0 paciente enquanto 0 medicamento está sob o controle do profissional de saúde, paciente ou consumidor. Tais eventos podem estar relacionados à prática profissional, produtos de cuidado de saúde, procedimentos, e sistemas, incluindo prescrição; comunicação; etiquetação, embalagem e nomenclatura; aviamento; dispensação; distribuição; administração; $\quad$ educação; monitoramento e uso". (NCCMERP, 1998 [online]).

Os dados provenientes de pesquisas mostram que os erros na medicação representam uma triste realidade no trabalho dos profissionais de saúde, com sérias conseqüências para pacientes e organização hospitalar, pois suas causas repercutem negativamente nos resultados institucionais face aos indicadores relevantes da qualidade da assistência prestada aos pacientes hospitalizados (CARVALHO, 2000).

O relatório "To err is human: building a safer health system" do Institute of Medicine (EUA) publicado em 1999, baseado em estudos realizados no Colorado, Utah e Nova York, aponta que das 33,6 milhões de internações realizadas no ano de 1997, em hospitais dos EUA, por volta de 44.000 a 98.000 americanos morreram devido a 
SILVA, Ana Elisa Bauer de Camargo; CASSIANI, Silvia Helena De Bortoli - Administração de medicamentos: uma visão sistêmica para o desenvolvimento de medidas preventivas dos erros na medicação. Revista Eletrônica de Enfermagem, v. 06, n. 02, p. 279-285, 2004. Disponível em www.fen.ufg.br

problemas causados por erros na medicação (KOHN et al., 2001).

Os resultados de um estudo realizado em dois hospitais terciários de grande porte, Brighan and Women's Hospital e Massachuchusetts General Hospital, nos Estados Unidos da América, apontou uma média de 6,5 eventos adversos ao medicamento para cada 100 internações, dos quais $28 \%$ poderiam ter sido prevenidos (BATES et al., 1995).

Os erros na medicação podem, muitas vezes, causar dano ao paciente e, segundo Bates (1996), cerca de 30\% dos danos durante a hospitalização estão associadas a erros na medicação, os quais trazem também sérias conseqüências econômicas às instituições de saúde. Estima-se um gasto de aproximadamente US\$ 4.700 por evento adverso de medicamento evitável ou por volta de US\$ 2,8 milhões, anualmente, em um hospital de ensino com 700 leitos. O custo anual de morbidade e mortalidade referente a erros na medicação, nos EUA, tem sido estimado em torno de US\$ 76,6 bilhões (BERWICK \& LEAPE, 1999; KOHN et al., 2001, ANDERSON, 2002).

Os erros descritos tanto na literatura nacional como na internacional são tipados conforme descrição a seguir (RIBEIRO, 1991; DRAFT, 1992; CASSIANI, 1998, NCCMERP, 1998):

- Erros de omissão: qualquer dose nãoadministrada até o próximo horário de medicação.

- Erros na administração de um medicamento não-autorizado: administração de um medicamento ou dose de medicamento não-prescrito pelo médico.

- Erros em dose extra: administração de uma ou mais unidades de dosagem, além daquela prescrita.

- Erros referentes à via: administração pela via errada ou por uma via que não a prescrita.

- Erros com a dosagem: administração do medicamento em dosagens diferentes daquelas prescritas pelo médico.
- Erros devido ao horário incorreto: administrar medicamento fora dos horários predefinidos pela instituição ou da prescrição.

- Erros devido ao preparo incorreto do medicamento: medicamento incorretamente formulado ou manipulado: diluição ou reconstituição incorreta ou inexata; falha ao agitar suspensões; diluição de medicamentos que não permitam esse procedimento, mistura de medicamentos que são física ou quimicamente incompatíveis e embalagem inadequada do produto.

- Erros devido à utilização de técnicas incorretas na administração: uso de procedimentos inconvenientes ou técnicas impróprias, como falhas nas técnicas de assepsia e das lavagens das mãos.

- Erros com medicamentos deteriorados: administração de medicamentos com comprometimento da integridade física ou química.

- Outros tipos de erros incluem:

- Erros de prescrição: prescrição imprópria de um medicamento, seja em relação à dose, apresentação, quantidade, via de administração ou concentração.

- Erros de distribuição: falhas ao distribuir o medicamento, como: doses incorretas; rótulos incorretos ou inadequados; preparação incorreta ou inapropriada; distribuição de medicamento com data expirada; medicamento estocado de maneira imprópria ou ainda comprometido física ou quimicamente.

- Erros potenciais: são aqueles que ocorreram na prescrição, distribuição ou administração dos medicamentos, mas que não causaram dano ao paciente.

Medicar pacientes requer um processo de comunicação eficaz para que esta atividade seja realizada com sucesso. Problemas na comunicação podem ser uma das causas de erros na medicação, e se 
SILVA, Ana Elisa Bauer de Camargo; CASSIANI, Silvia Helena De Bortoli - Administração de medicamentos: uma visão sistêmica para o desenvolvimento de medidas preventivas dos erros na medicação. Revista Eletrônica de Enfermagem, v. 06, n. 02, p. 279-285, 2004. Disponível em www.fen.ufg.br

originam de várias situações encontradas no dia-a-dia do profissional (BARKER \& HELLER, 1964 apud RIBEIRO, 1991, p. 70). Dentre essas situações, a má qualidade da letra médica é identificada como um fator que contribui não só para a incidência de erros, como também para aumentar o custo para o hospital, pois exige mais tempo da enfermagem e dos profissionais da farmácia para interpretá-la. A falta de uma padronização da nomenclatura de medicamentos também pode gerar problemas, pois muitos deles possuem nomes comerciais parecidos, podendo ser transcritos ou interpretados de maneira incorreta. Além disso, o uso de abreviaturas nas prescrições, padronizadas ou não, podem originar erros.

As ordens verbais são freqüentes na prática hospitalar, principalmente em situações de emergência, porém exigem uma boa memória do receptor para guardar a dose e nome do medicamento indicado, pois muitas vezes elas são dadas de forma incompleta. No caso das prescrições escritas, as informações podem ser também incompletas e faltar dados como: via, dose, posologia, diluição, levando o profissional a tirar conclusões próprias sobre como agir, seja executando ou deixando de realizar o procedimento.

Em alguns hospitais é comum o ato de transcrições das ordens médicas que podem induzir à omissão ou erro na transmissão da informação.

Os erros na medicação também podem estar relacionados: a deficiências da formação acadêmica, inexperiência, negligência, desatenção ou desatualização quanto aos avanços tecnológicos e científicos; ao manejo de equipamentos, como bombas de infusão, cateteres etc; aos procedimentos desenvolvidos e ao sistema de medicação como um todo.

Sabe-se que os erros fazem parte da natureza humana, portanto os sistemas de medicação devem ser bem estruturados com a finalidade de promover condições que auxiliem na minimização e prevenção dos erros, planejando os processos e implementando normas, regras e ações.

Alguns estudos têm como propósitos identificar e analisar os erros na medicação, mas o fazem sob a ótica dos erros causados pelos profissionais, ignorando que erros na medicação também podem ser gerados por falhas no sistema que, muitas vezes, induzem ao erro humano (BRENNAM, 1991; PHELAN, 1996).

No caso do erro na medicação, o indivíduo raramente é a única causa. Desse modo, entendemos que deve-se mudar a cultura dos erros para que estes não sejam vistos como falhas humanas, mas como uma oportunidade de melhorar o sistema existente (BERWICK et al, 1994; LEAPE, 1994; PEPPER, 1995).

No momento em que a instituição procurar saber "como" e o "por quê" da ocorrência dos erros, e não mais o "quem" foi o responsável por eles, ela contará com a colaboração dos profissionais para diagnosticar as falhas que possam estar ocorrendo na medicação dos pacientes.

Sabemos que o medo de ações disciplinares e punitivas compromete a decisão do funcionário ou da equipe quanto a documentá-lo ou não, portanto, os gerentes de serviços devem mudar sua cultura e promover mudanças no ambiente de trabalho, de modo que a punição não seja a prática em execução e o ser humano não seja o foco principal de correção (CASSIANI, 1998; CASSIANI, 2000b).

Os gerentes muitas vezes pensam que, sem punição, os profissionais se tornarão descuidados e com isso os erros poderão aumentar, mas WOMER et al. (2002), em estudo realizado sobre a prática da nãopunição no Children's Hospital of Philadelphia, identificaram que os relatos de erros interceptados aumentaram dramaticamente enquanto os dos detectados diminuíram.

\section{O SISTEMA DE MEDICAÇÃO}

O conceito de sistema traz as relações entre as partes e o todo, permitindo a 

medicamentos: uma visão sistêmica para o desenvolvimento de medidas preventivas dos erros na medicação. Revista Eletrônica de Enfermagem, v. 06, n. 02, p. 279-285, 2004. Disponível em www.fen.ufg.br

compreensão de toda e qualquer atividade complexa, sendo os sistemas constituídos de conjuntos de componentes que se interagem, se inter-relacionam, se transformam e atuam entre si na execução de um objetivo global. Estes conjuntos poderão ser assumidos como subsistemas ou processos, com funções e objetivos próprios, os quais afetam o comportamento do conjunto como um todo. Qualquer ação de uma parte, necessariamente, provocará uma reação das demais (CHURCHMAN, 1975; MELO, 1987; ACKOFF apud ARAÚJO, 1994).

O sistema de medicação de um hospital é aberto e complexo, envolvendo várias etapas que estão inter-relacionadas e interligadas por várias ações, desenvolvendo de 20 a 30 passos diferentes durante os processos de prescrição, dispensação e administração de medicamentos, envolvendo sempre muitos indivíduos e múltiplas transferências de pedidos ou materiais, que passam de uma mão à outra, que podem conduzir a erros na medicação (LEAPE et al., 2000).

Em 1989, a Joint Commission on Accreditation of Healthcare Organizations JCAHO identificou cinco componentes ou processos do sistema de medicação, quais sejam: seleção e obtenção do medicamento; prescrição; preparo e dispensação; administração de medicamentos e acompanhamento do paciente em relação aos efeitos do medicamento (NADZAM, 1998).

Estudo realizado por LEAPE et al. (1995) relata que $39 \%$ dos erros na medicação ocorreram no processo de prescrição de medicamentos, $12 \%$ na transcrição, $11 \%$ no processo de dispensação e 38\% no de administração de medicamentos.

No sistema de medicação entendemos por insumo os pacientes e as informações a respeito da terapia medicamentosa; por processo, a realização de prescrição, dispensação e distribuição e administração de medicamentos; por resultado, os pacientes medicados com eficácia, e o tratamento seguro, como feed-back, que realimenta o sistema, os relatórios e as informações geradas. Por último, temos como ambiente $o$ conjunto de fatores $e$ de condições que influencia a dinâmica desta prática (tecnologia, infra-estrutura, fornecedores, regras, regulamentos etc) fazendo com que o sistema se reajuste e se mantenha em contínua aprendizagem e autoorganização (NADZAM, 1998).

Os médicos são os responsáveis pela prescrição de medicamentos, porém a maneira como ela é realizada varia de hospital para hospital. A equipe de enfermagem atua no último processo, que é o da administração do medicamento ao paciente, fato que faz com que muitos erros cometidos no início ou no meio do sistema, e não detectados, Ihe sejam atribuídos.

As instituições hospitalares devem prevenir os erros na medicação através da elaboração e implementação de mecanismos de segurança voltados ao sistema de medicação, deixando de lado a perseguição e punição dos profissionais a cada erro ocorrido, fazendo com que este erro seja discutido e, assim, revertido em educação e melhorias para o sistema. Para tanto o sistema deve possuir alguns princípios e características considerados ideais (LEE, 2002).

Os princípios ideais apregoam que o sistema deva centrar-se no paciente, basearse no respeito aos outros e requerer o aceite à responsabilidade e à colaboração de todos os interessados, tanto dos profissionais envolvidos como do próprio paciente que também deve assumir responsabilidade de participar das decisões relativas ao seu tratamento.

Em um estudo multidisciplinar foram utilizados alguns princípios de segurança e orientações que resultaram em melhorias para o sistema, reduzindo os erros que atingiam o paciente, tais como (WOMER et al., 2002): eliminar as punições; aprender com o erro; simplificar o sistema de medicação; padronizar os processos; utilizar procedimentos que estimulem as pessoas a seguirem o caminho certo; reduzir a transferência de atividades entre pessoas 
SILVA, Ana Elisa Bauer de Camargo; CASSIANI, Silvia Helena De Bortoli - Administração de medicamentos: uma visão sistêmica para o desenvolvimento de medidas preventivas dos erros na medicação. Revista Eletrônica de Enfermagem, v. 06, n. 02, p. 279-285, 2004. Disponível em www.fen.ufg.br

e/ou grupos de pessoas; dar principal atenção ao fator humano.

Além da complexidade própria do sistema de medicação, os hospitais ligados às Universidades, que oferecem campo de estágio para escolas técnicas e que possuem acadêmicos e estudantes das diversas áreas da saúde atuando em suas instalações e prestando assistência a seus pacientes, devem considerar as situações inerentes àqueles que estão aprendendo, tais como: inabilidade, insegurança, falta de domínio técnico, limitada capacidade para interpretar e analisar as situações presentes e emergentes, precipitação, lentidão, apresentando, enfim, possibilidade maior de risco para a falha e para a iatrogenia do cuidado (MADALOSSO, 2000). Acrescentase ainda a esse processo o pouco conhecimento dos sistemas vigentes na instituição e seu papel dentro deles.

Este fato pode prejudicar a qualidade e a eficiência dos serviços prestados aos pacientes, principalmente no que se refere ao sistema de medicação que sempre comporta riscos, podendo, assim, causar danos à clientela.

\section{CONSIDERAÇÕES FINAIS}

As instituições hospitalares devem centrar seus objetivos em um sistema de medicação seguro aos pacientes, utilizando, para este fim: mudança na cultura dos erros; simplificação e padronização dos processos e as atividades neles desenvolvidos e introdução de avaliações imediatas sobre as ações executadas.

O Joint Commission on Accreditation of Healthcare Organizations faz algumas recomendações gerais para prevenção de erros como: reduzir a confiança na memória; aperfeiçoar o acesso a informações seguras e confiáveis sobre medicamentos; introduzir sistemas que eliminem ou diminuam a possibilidade de erro (ex: equipo de nutrição enteral que não conecte em cateter intravenoso); buscar a padronização e promover treinamentos (BATES, 1998).
Percebemos que a maioria das estratégias está voltada ao sistema e sabemos que prevenir erros através de abordagens voltadas para os indivíduos que os cometem é contraproducente, pois tais estratégias podem encorajar o funcionário a esconder os erros com medo das possíveis punições e da exposição a que estará sujeito.

Em vista disso, torna-se necessário conhecer o sistema de medicação utilizado nas instituições hospitalares e verificar como seus profissionais estão desenvolvendo suas atividades, se estas são preestabelecidas ou não por protocolos e padronizações, para permitirem identificação e análise das possíveis fragilidades e falhas ocorridas nos processos e intervir, minimizando riscos e aumentando a segurança do paciente.

\section{REFERÊNCIAS}

ANDERSON, J. G. et al. Evaluating the capability of information technology to prevent adverse drug events: a computer simulation approach. J. Am. Med. Inform Assoc., v. 9, n. 5, p. 479-490, 2002.

ARAUJO, L. C. G. Organização \& métodos: integrando comportamento, estrutura, estratégia. São Paulo: Atlas, 1994.

BATES, D. W. Preventing medication errors. In: COUSINS, D. M. Medication use: a system approach to reducing errors. Oakbrook Terrace: Joint Commission on Accreditation of Healthcare Organizations, 1998. p.57-73.

BATES, D. W. et al. Incidence of adverse drug events and potential adverse drug events: implications for prevention. JAMA, v. 274, n. 1, p.29-34, 1995.

BERWICK, D. M.; LEAPE. L. L. Reducing errors in medicine. $B M J$, v. 319 , p. $136-137$, 1999.

BERWICK, D. M. et al. Melhorando a qualidade dos serviços médicos, hospitalares e da saúde. Trad. de José Carlos Barbosa dos Santos. São Paulo: Makron Books, 1994. BRENNAM, T. A. et al. Incidence of adverse events and negligence in hospitalized patients. N. Engl. J. Med., v. 324, n. 6, p. 370376, 1991. 
SILVA, Ana Elisa Bauer de Camargo; CASSIANI, Silvia Helena De Bortoli - Administração de medicamentos: uma visão sistêmica para o desenvolvimento de medidas preventivas dos erros na medicação. Revista Eletrônica de Enfermagem, v. 06, n. 02, p. 279-285, 2004. Disponível em www.fen.ufg.br

CARVALHO, V. T. Erros na administração de medicamentos: análise dos relatados dos profissionais de enfermagem. Ribeirão Preto, 2000. 139f. Dissertação (Mestrado) - Escola de Enfermagem de Ribeirão Preto, Universidade de São Paulo.

CASSIANI, S. H. B. Um salto para o futuro no ensino da administração de medicamentos: desenvolvimento de um programa instrucional auxiliado pelo computador. Ribeirão Preto, 1998. 206 p. Tese (Livre Docência) - Escola de Enfermagem de Ribeirão Preto, Universidade de São Paulo.

CASSIANI, S. H. B. Erros na medicação: Estratégias de Prevenção. Rev. Bras. Enf., v. 53, n. 3, p. 424-430, 2000.

CHURCHMAN, C. W. Introdução à teoria dos sistemas. $2^{a}$ ed. Petrópolis: Vozes, 1975.

DRAFT Guidelines on preventable medication errors. Am. J. Hosp. Pharm., v. 49, n. 3, p. 640-648, 1992.

$\mathrm{KOHN}, \mathrm{L}$. T. et al. To Error is human: building a safer health system. Washington: Committee on Quality of Health Care in America, National Academy of Institute of Medicine, 2001.

LEAPE, L. L. Error in medicine. JAMA, v. 272, n. 23, p.1851-7, 1994.

LEAPE, L. L. et al. Systems analysis of adverse drug events. JAMA, v. 274, n.1, p. 35-43, 1995.

LEAPE, L. L. et al. Reducing adverse drug events: lessons from a breakthroughs series collaborative. Jt. Comm. J. Qual. Improv., v. 26, n. 6 p. 321-331, 2000.

LEE, P. Ideal principles and characteristics of a fail-safe medication-use system. Am. J. Health-System Pharm., v. 59, n. 4, p. 369371, 2002.

MADALOSSO, A. R. M. latrogenia do cuidado de enfermagem: dialogando com o perigo no quotidiano profissional. Rev. Latino - Am. Enf, v. 8, n. 3, p. $11-17,2000$.

MELO, I. S. Sistemas de informação. $3^{a}$ ed. São Paulo: Saraiva, 1987.

NADZAN, D. M. A system approach to medication use. In: COUSINS, D. M. Medication use: a system approach to reducing errors. Oakbrook Terrace: 1998. p. 5-18.

NCCMERP - NATIONAL COORDINATING COUNCIL FOR MEDICATION ERROR REPORTING AND PREVENTION. Taxonomy of medication errors. , 1998. Disponível em $<$ http://www.nccmerp.org/public/aboutmederr or.html> Acesso em 10 fev. 2003

PEPPER G. A. Errors in drug administration by nurses from Understanding and Preventing Drug Misadventures Conference. Am. Soc. Health-System Pharm., v. 52, n. 4, p. $390-$ 395, 1995.

PHELAN, G. et al. Self - administration of medication by patients and family members during hospitalization. Patient Educ. Couseling, v. 27, p. 103-112, 1996.

RIBEIRO, E. Dose Unitária - Sistema de distribuição de medicamentos em hospitais. São Paulo, 1991. 476 p. Dissertação (Mestrado) Escola de Administração Hospitalar e de Sistemas de Saúde da Fundação Getúlio Vargas.

TAXIS, K.; BARBER, N. Ethnographic study of incidence and severity of intravenous drug errors. BMJ, v. 326, n. 7391, p. 684-687, mar. 2003.

WOMER, R. B. et al. Multidisciplinary systems approach to chemotherapy and holding the gains. J. Clin. Oncol., v. 20, n. 24, p. 4705-4712, dec. 2002.

Texto recebido em 30/03/2004

Publicação aprovada em 30/04/2004 